\title{
Philosophiques
}

\section{Remarques sur la sémiotique}

\section{Daniel Laurier}

Volume 11, numéro 1, avril 1984

URI : https://id.erudit.org/iderudit/203242ar

DOI : https://doi.org/10.7202/203242ar

Aller au sommaire du numéro

Éditeur(s)

Société de philosophie du Québec

ISSN

0316-2923 (imprimé)

1492-1391 (numérique)

Découvrir la revue

Citer cet article

Laurier, D. (1984). Remarques sur la sémiotique. Philosophiques, 11(1), 91-109. https://doi.org/10.7202/203242ar

\section{Résumé de l'article}

Je défends la classification carnapienne des disciplines sémiotiques en montrant qu'elle permet de caractériser adéquatement la nature de la pragmatique. J'indique, en particulier, comment une notion de système pragmatique pourrait être développée par analogie avec celles de système syntaxique et de système sémantique. d'utilisation que vous pouvez consulter en ligne.

https://apropos.erudit.org/fr/usagers/politique-dutilisation/ 


\title{
REMARQUES SUR LA SÉMIOTIQUE
}

\author{
par Daniel Laurier
}

\begin{abstract}
RÉSUMÉ. Je défends la classification carnapienne des disciplines sémiotiques en montrant qu'elle permet de caractériser adéquatement la nature de la pragmatique. J'indique, en particulier, comment une notion de système pragmatiqué pourrait être développée par analogie avec celles de système syntaxique et de système sémantique.
\end{abstract}

ABSTRACT. I defend Carnap's classification of semiotic disciplines by showing that it leads to an adequate characterization of the nature of pragmatics. In particular, I indicate how a notion of pregmatic system could be constructed on the analogy with those of syntactic system and semantic system.

\section{LA TRICHOTOMIE MORRISSIENNE}

Une tradition ancienne mais respectable veut qu'un système linguistique soit entièrement caractérisé par la donnée du système des règles syntaxiques qui en définit l'ensemble des expressions bien formées et du système des règles sémantiques qui associe à chacune d'elles un élément de l'univers du discours, qui constitue sa dénotation. Dans cette perspective "augustinienne ", il est naturel d'identifier la signification d'une expression à sa dénotation et de considérer que l'énoncé de ces deux systèmes de règles, syntaxiques et sémantiques, constitue la description grammaticale complète de tout système linguistique possible.

Le trait le plus remarquable d'une telle conception du langage est sans doute qu'elle néglige totalement le fait qu'un système linguistique puisse être utilisé, que ce soit pour communiquer, pour raisonner, pour imaginer des états de choses plus ou moins probables ou simplement pour exprimer ses désirs ou ses craintes ; elle laisse donc dans l'ombre la question des 
relations entre un système linguistique et ses utilisateurs réels ou possibles et reste exclusivement sur le terrain de la logique et/ou de l'ontologie. Or il semble incontestable que la description d'un mécanisme quelconque, qu'il s'agisse d'une langue, d'un ordinateur ou d'une locomotive, est incomplète si elle ne fait pas mention de son fonctionnement, mais seulement de sa structure.

Le terme de "pragmatique » a été introduit par Charles Morris dans les années trente pour désigner l'étude des relations entre les systèmes linguistiques et leurs utilisateurs. Cette définition nominale laisse manifestement place à une grande variété de programmes de recherche. Un système linguistique est en effet utilisé (utilisable) par certains organismes (ou sujets) qui ont telle ou telle structure interne, pour remplir telles ou telles fonctions de telle ou telle manière ; c'est dire qu'on ne peut raisonnablement parler de l'utilisation d'un système linguistique que relativement à une classe d'utilisateurs, une classe de fonctions linguistiques (ou mieux : communicatives) et une classe de stratégies ou règles d'utilisation qui rendent compte de la façon dont ces fonctions sont remplies. N'importe quel travail de description ou d'explication de la façon dont un système linguistique ou une famille de systèmes linguistiques sont conceptuellement ou empiriquement reliés à quelques-uns ou à chacun de ces trois paramètres relèverait donc plus ou moins d'une pragmatique.

Cependant, il semble être généralement admis (au moins tacitement) que quelque soit le contenu effectif qu'il faille finalement donner à ce terme, la pragmatique ne détermine pas un niveau supplémentaire de la description grammaticale, à mettre sur le même pied que la description syntaxico-sémantique, de sorte que la conception "augustinienne " évoquée plus haut n'est pas réfutée mais seulement complétée par l'avènement de la pragmatique. Si on cherchait une analogie avec d'autres disciplines, c'est bien évidemment de la physiologie plutôt que de l'anatomie qu'il faudrait rapprocher la pragmatique, au moins dans sa visée. Le " passage au pragmatique " implique la prise en considération, dans la description des phénomènes linguistiques, de leurs aspects fonctionnels et diachroniques (non pas " historiques » mais pour ainsi dire " micro-diachroniques"). 
La pragmatique embrasse donc un vaste domaine qu'il convient d'organiser minimalement. Carnap (1942) reprend la tripartition morrissienne de la sémiotique en syntaxe, sémantique et pragmatique, et partage en outre les disciplines sémiotiques en pures ou descriptives d'une part, et générales ou spéciales d'autre part. Cette combinatoire prévoit ainsi une syntaxe (sémantique, pragmatique) générale pure, une syntaxe (sémantique, pragmatique) générale descriptive, des syntaxes (sémantiques, pragmatiques) spéciales pures et des syntaxes (sémantiques, pragmatiques) spéciales descriptives. J'indiquerai ici brièvement comment ces distinctions me semblent pouvoir être interprétées.

\section{SYNTAXES ET SÉMANTIQUES}

On conviendra aisément que la sémiotique est l'étude des propriétés des systèmes sémiotiques (linguistiques). On dira ainsi que la syntaxe étudie les systèmes syntaxiques, la sémantique les systèmes sémantiques et la pragmatique les systèmes pragmatiques.

\subsection{Syntaxes et sémantiques pures}

Dans ces conditions, l'objet de la syntaxe générale pure est d'étudier les propriétés générales communes à tous les systèmes syntaxiques possibles et donc de caractériser l'ensemble des systèmes syntaxiques possibles; de façon équivalente, la syntaxe générale pure doit expliquer/analyser le concept de système syntaxique, ce qui implique aussi l'élaboration des concepts théoriques fondamentaux de la syntaxe (ceux de concaténation, de règle et de catégorie syntaxiques etc . . .). De même, la sémantique générale pure devrait caractériser l'ensemble des systèmes sémantiques (ou modèles) possibles et ainsi définir les notions de règle et de catégorie sémantiques, de vérité, de dénotation, etc... ${ }^{1}$.

1. Bien que les notions de théorème et de démonstration soient habituellement comptées au nombre des notions purement syntaxiques ou formelles, elles ne concernent pas à proprement parler la syntaxe générale pure, mais plutôt la syntaxe logique. Ces notions n'ont de sens que dans la perspective d'une formalisation de la logique ; elles ne valent que comme des explications "syntaxiques" de phénomènes essentiellement sémantiques. Elles reposent sur la notion de règle d'inférence, qui est elle-même ultimement gouvernée par celle de vérité. 
Chaque interprétation possible d'un système syntaxique SY peut être conçue comme une certaine relation $f$ entre les expressions de SY et les éléments d'un certain ensemble d'objets $M$; en ce sens on peut dire que l'ensemble des systèmes sémantiques possibles est compris dans l'ensemble des couples $<\mathrm{f}, \mathrm{M}>$ pour lesquels le domaine de $f$ est un système syntaxique possible et son champ est inclus dans $M .{ }^{2}$ Il est naturellement essentiel que cet ensemble ne soit ni vide ni universel, s'il doit être consistant et significatif de parler de modèles d'un système syntaxique ; la fonction $\mathrm{f}$ sera donc soumise à certaines contraintes qu'il revient à la théorie sémantique de préciser.

La sémantique générale pure doit donc, notamment, formuler les conditions minimales que doivent satisfaire tout univers de discours et toute assignation de dénotations possible pour un système syntaxique quelconque ; en d'autres termes, le concept de système sémantique possible doit être défini de telle façon que la structure de tout modèle possible soit fonction de celle du système syntaxique dont il est un modèle. Comme tout système sémantique possible est un modèle d'un système syntaxique possible et d'un seul, on voit que la sémantique générale pure ne se distingue guère de ce qu'on pourrait appeler la grammaire générale pure (i.e. l'étude «structurale » des systèmes linguistiques) puisqu'elle permet de définir l'ensemble des systèmes linguistiques possibles comme étant l'ensemble des couples $<\mathrm{SY}<\mathrm{f}, \mathrm{M}>>$ tels que SY est le domaine de $\mathrm{f}$ et $<\mathrm{f}, \mathrm{M}>$ est un système sémantique. À chaque système sémantique possible correspond donc un et un seul système linguistique et vice-versa. Ceci confirme simplement l'intuition que la sémantique englobe la syntaxe, et qu'il n'est pas possible de détacher un système sémantique du système syntaxique dont il est un modèle (ou mieux, une interprétation). On parlera donc de l'interprétation d'un système linguistique, mais des interprétations (ou d'une interprétation) d'un système syntaxique.

Toute définition de la notion d'interprétation qui impliquerait que certains systèmes syntaxiques n'admettent aucune

2. Le fait que les modèles pour les langages intensionnels contiennent habituellement des mondes possibles ou autres genres d'indices ne révèle pas l'insuffisance de cette notion de système sémantique ; cela montre seulement que l'univers du discours peut être construit à partir d'autres ensembles. 
interprétation, c'est-à-dire que l'ensemble des systèmes syntaxiques interprétables soit strictement compris dans celui des systèmes syntaxiques possibles, serait sans doute jugée inadéquate. Si une telle définition était proposée on aurait certainement tendance à croire soit qu'elle est trop restrictive soit que la notion de système syntaxique acceptée jusque là ne l'était pas assez. Cela vient probablement de ce que la notion de système linguistique avec laquelle nous opérons en fait, prévoit par exemple que tout couple de la forme $=, S Y$ où le domaine de la relation d'identité " = " est le système syntaxique SY, constitue une interprétation possible de SY. Cela est d'autant plus remarquable qu'on ne peut en dire autant de la notion de système pragmatique possible, que la pragmatique générale pure devrait en toute logique caractériser : il pourrait se faire qu'une définition adéquate de la notion de système pragmatique ait pour conséquence que certains systèmes linguistiques possibles ne sont pas utilisables.

Chaque syntaxe ou sémantique spéciale pure définit un certain type de systèmes syntaxiques ou sémantiques possibles et étudie les propriétés propres à tous les systèmes de ce type. En d'autres termes, la syntaxe et la sémantique pures sont des sciences logico-mathématiques, purement déductives.

\subsection{Syntaxes et sémantiques descriptives}

La syntaxe (sémantique) générale descriptive devrait alors être l'étude des systèmes syntaxiques (sémantiques) empiriquement (ou historiquement) donnés ; elle devrait caractériser l'ensemble des systèmes syntaxiques (sémantiques) effectivement réalisés, qui serait lui-même compris dans l'ensemble des systèmes syntaxiques (sémantiques) possibles défini par la syntaxe (sémantique) générale pure (à moins que les concepts de la syntaxe ou de la sémantique générales pures ne se révèlent être trop restrictifs, ce qui ne saurait être exclu a priori).

Il faut toutefois remarquer que cet ensemble ne peut être univoquement caractérisé qu'à condition soit d'en énumérer les éléments, soit de considérer le fait d'être empiriquement réalisé comme une propriété supplémentaire que tous les systèmes syntaxiques (sémantiques) réalisés (i.e. existants) auraient en com- 
mun ; mais dans ce cas il n'est pas nécessaire de mentionner d'autres propriétés que les systèmes syntaxiques (sémantiques) réalisés auraient en commun pour en définir l'ensemble. Aucun de ces procédés n'apporte d'information sur les systèmes syntaxiques (sémantiques) réalisés. D'autre part, il est inévitable que, quelles que soient les propriétés communes propres aux systèmes syntaxiques (sémantiques) réalisés, l'ensemble des systèmes syntaxiques (sémantiques) possédant ces propriétés ne contienne pas que des systèmes syntaxiques (sémantiques) effectivement réalisés.

On ne peut donc espérer caractériser univoquement, au moyen des concepts de la syntaxe (sémantique) que le plus petit ensemble de systèmes syntaxiques (sémantiques) possibles à contenir tous les systèmes syntaxiques (sémantiques) réalisés. En d'autres termes, la syntaxe (sémantique) générale descriptive ne pourra jamais formuler que les conditions nécessaires à la réalisation d'un système syntaxique (sémantique); aussi sévères que soient ces conditions, elles ne définiront jamais exactement l'ensemble des systèmes syntaxiques (sémantiques) réalisés à moins de contenir une longue disjonction de propriétés dont chacune corresponde à un système syntaxique (sémantique) réalisé et un seul et vice-versa (c'est-à-dire, à moins d'être équivalente à une énumération). Il n'est donc pas possible de maintenir que la syntaxe (sémantique) générale descriptive doit définir ou caractériser l'ensemble des systèmes syntaxiques (sémantiques) réalisés ; cela n'est d'ailleurs pas requis pour qu'elle(s) puisse(nt) être considérée(s) comme une (des) science(s) empirique(s).

Un problème analogue se pose aussi au niveau de la syntaxe (sémantique) pure, qui explique que tout sous-ensemble de systèmes syntaxiques (sémantiques) possibles ne constitue pas le domaine d'une syntaxe (sémantique) spéciale pure. Cela tient au fait qu'une syntaxe (sémantique) spéciale pure qui étudierait les propriétés de tel système syntaxique (sémantique) particulier doit se confondre soit avec la donnée de ce système lui-même (puisque la définition d'un système syñtaxique et a fortiori sémantique, implique l'énumération de ses symboles primitifs, i.e. l'usage de termes singuliers), soit avec la syntaxe (sémantique) spéciale pure qui prend aussi pour objet tout autre système qui ne differe du premier que par ses symboles primitifs. 
En d'autre termes, le plus petit ensemble de systèmes syntaxiques (sémantiques) possibles définissables à l'aide des seuls concepts théoriques (ou prédicats) de la syntaxe (sémantique) comprend tous les systèmes " isomorphes " à (i.e. qui sont des variantes alphabétiques d') un système donné, et ceux-ci sont en nombre infini.

D'autre part, quel que soit l'ensemble de systèmes syntaxiques (sémantiques) caractérisé par une syntaxe (sémantique) descriptive (i.e. le plus petit ensemble de systèmes syntaxiques (sémantiques) possibles définissable en termes généraux, à contenir tous les ou certains systèmes syntaxiques (sémantiques) réalisés, selon qu'on considère la syntaxe (sémantique) générale descriptive ou une syntaxe (sémantique) spéciale descriptive), il coïncidera avec le domaine d'une syntaxe (sémantique) pure. La question se pose donc de savoir ce qui distingue une syntaxe (sémantique) descriptive donnée de la syntaxe (sémantique) pure qui étudie le même ensemble de systèmes syntaxiques (sémantiques) possibles.

On a partiellement répondu à cette question en soulignant le caractère déductif de la sémantique pure; mais peut-être le fait de parler des syntaxes (sémantiques) pures comme devant définir des types de systèmes syntaxiques (sémantiques) ne faitil pas assez ressortir qu'il s'agit moins de définir des types de structures que d'en étudier les propriétés formelles. Le problème de la syntaxe (sémantique) descriptive est tout différent, et consiste à établir une correspondance entre les structures définies par la syntaxe (sémantique) pure et certains phénomènes empiriques. En d'autres termes, l'opposition du pur au descriptif reproduirait celle du conceptuel (théorique) à l'empirique (observationnel).

Au sens strict, ce ne sont pas des systèmes linguistiques qui sont réalisés, mais seulement certaines de leurs expressions (ou des occurrences de ces expressions) qui sont les seules données empiriques sur lesquelles on puisse reconstruire les systèmes syntaxiques et sémantiques dont elles relèvent. C'est dire que même l'idée qu'un certain ensemble de formes ou d'expressions fait partie de tel système linguistique possible n'est jamais qu'une hypothèse empirique, et que les propriétés formelles des sys- 
tèmes linguistiques mises au jour par la syntaxe et la sémantique pures sont appelées à jouer un rôle essentiel dans l'identification des systèmes linguistiques empiriquement manifestés (de même que la physique mathématique joue un rôle dans la corroboration des théories physiques). C'est à la syntaxe et à la sémantique pures que la syntaxe et la sémantique descriptives empruntent nécessairement l'appareil conceptuel et déductif qui leur confere leur force explicative et leur caractère nomologique. Si on pouvait imaginer identifiés/reconstruits tous les systèmes syntaxiques dont on a pu repérer des manifestations empiriques, il n'y aurait rien pour distinguer la partie théorique de la syntaxe (sémantique) générale descriptive de la syntaxe (sémantique) pure correspondante. La syntaxe (sémantique) pure épuise ainsi le contenu théorique de la syntaxe (sémantique) descriptive.

En pratique cependant, la syntaxe (sémantique) générale descriptive servira souvent de guide à la reconstruction des systèmes linguistiques réalisés particuliers en excluant plus ou moins a priori certaines possibilités, et jouera ainsi un rôle méthodologique essentiel. Plus exactement, il y a une interaction constante au niveau descriptif entre syntaxe (sémantique) générale et syntaxes (sémantiques) spéciales : une décision prise (une hypothèse formulée) au niveau de la syntaxe (sémantique) générale descriptive aura des répercussions au niveau des syntaxes (sémantiques) spéciales descriptives et forcera éventuellement la répudiation d'hypothèses plus particulières ; et inversement les hypothèses formulées au niveau de la description des systèmes linguistiques particuliers pourront remettre en question celles de la syntaxe (sémantique) générale descriptive.

\section{PRAGMATIQUES}

Ceci montre, je pense, que les deux couples d'opposition carnapiens du pur au descriptif et du général au spécial sont applicables à la syntaxe et à la sémantique. Mais la combinatoire de Carnap distingue aussi une pragmatique générale pure, dont la tâche devrait être, si l'analogie avec la syntaxe et la sémantique doit être maintenue, d'expliquer le concept de système pragmatique possible et d'étudier les propriétés formelles des structures ainsi définies. Nous avons une certaine intuition de ce que c'est pour une expression linguistique d'être bien formée ou de 
dénoter un objet donné et les notions de système syntaxique et de système sémantique ne sont, en un sens, rien d'autre que des constructions destinées à rendre compte de ces intuitions. De la même façon, nous avons une certaine idée de ce que c'est qu'utiliser une expression linguistique, dont l'analyse devrait permettre de construire la notion de système pragmatique.

\subsection{Notion de système pragmatique}

On a déjà remarqué que la notion d'utilisation d'un système linguistique renvoyait à celles d'utilisateur, de fin ou fonction, et de règles d'utilisation. On peut donc croire, en première approximation, que chaque système pragmatique possible sera déterminé par le choix d'un système linguistique, d'un type d'utilisateurs, d'un ensemble de «fonctions » et d'un ensemble de règles d'utilisation. Le problème consisterait à analyser chacune de ces notions et à en préciser les interrelations ; c'est-àdire à déterminer les contraintes que le choix d'un de ces quatre éléments place sur celui de chacun des trois autres.

\subsubsection{L'utilisation d'un système linguistique}

Bien qu'à un certain niveau l'utilisation d'un système linguistique consiste essentiellement à produire et à recevoir des (suites d') expressions linguistiques, la fonction des règles d'utilisation d'un système linguistique n'est pas d'expliquer comment un utilisateur peut reconnaître et/ou réaliser des expressions linguistiques (ce qu'on peut probablement abandonner à la psychophysiologie) ; elles doivent déterminer ce qu'il peut faire ou vouloir faire en produisant des expressions linguistiques; en d'autres termes elles doivent préciser quelle est la fonction propre de chaque énonciation.

Le fait de produire une expression linguistique dans telles ou telles conditions lui confere une valeur fonctionnelle et représentationnelle spécifique, qu'il revient aux règles d'utilisation (moyennant certaines prémisses auxiliaires) de préciser, et qui dépend essentiellement de l'existence d'une communauté d'utilisateurs partageant un certain nombre de croyances, de pratiques et de valeurs ou intérêts. Ceci ne signifie pas que les règles d'utilisation d'un système linguistique soient complètement 
déterminées par l'organisation sociale ou l'idéologie de la communauté considérée ; la structure du système linguistique donné et la psychologie des membres de cette communauté y contribuent aussi vraisemblablement.

Chaque système de règles d'utilisation expliquera donc comment la production de telle ou telle expression dans telles ou telles conditions affecte le statut réciproque de certains membres de la communauté linguistique considérée relativement au contenu de l'énoncé, aussi bien que ce contenu lui-même. En d'autres termes, il devra définir l'ensemble des (suites d') illocutions possibles eu égard à une certaine classe d'utilisateurs (« illocution» désigne ici le sens d'une énonciation complète).

L'étude de certains types d'effets perlocutoires, comme la persuasion, et en général tous ceux qui présupposent ou résultent de la performance d'une (suite d') illocution(s) appartient vraisemblablement aussi à la pragmatique, mais on peut croire qu'ils seront des conséquences de la théorie des illocutions en conjonction avec certains principes généraux d'une théorie de l'action. Dans la mesure où la théorie des perlocutions intéresse la pragmatique, elle est d'abord une théorie de l'utilisation des illocutions et ne concerne que dérivativement, et partiellement, l'utilisation des expressions linguistiques proprement dites. D'autre part, un effet perlocutoire est un effet pour ainsi dire « naturel » de l'illocution, en ce sens que sa réalisation est indépendante aussi bien des conventions linguistiques de la communauté que des intentions des utilisateurs.

La notion d'utilisation d'un système linguistique renvoie donc nécessairement à un espace d'intersubjectivité et les règles d'utilisation d'un système linguistique sont comparables à une (micro-) économie politique du discours, dans la mesure où elles correspondent aux lois de l'échange et de la valeur des expressions linguistiques dans une population donnée.

\subsubsection{La communauté linguistique}

Il est certainement difficile, voire impossible, d'imaginer quelles illocutions, ou plus vaguement quelles valeurs d'énonciation, pourraient être définies sans aucune référence à une communauté donnée de locuteurs-auditeurs. L'idée qu'il n'existe pas 
d'illocutions (ni donc d'énonciations) privées n'est cependant pas incompatible avec l'existence d'un système linguistique qui n'aurait en fait qu'un utilisateur. Elle exclut simplement que ce dernier puisse faire quoi que ce soit d'autre, en produisant des expressions linguistiques, que des actes locutoires (à moins de faire implicitement référence à une communauté d'utilisateurs possibles de ce système linguistique). Il semblerait que l'assertion et la supposition, par exemple, puissent fonctionner comme des expressions illocutoires privées ; mais alors il faudrait admettre qu'il n'y a aucune distinction entre le fait de juger (ou d'imaginer) que $p$ et le fait d'affirmer (ou de supposer) que $p$, ce qui n'est intuitivement pas le cas. S'il n'est pas nécessaire de s'adresser à un auditoire pour affirmer que $\mathrm{p}$, l'assertion de $\mathrm{p}$ implique cependant la réalisation dans un espace public, d'une forme symbolique signifiant que $p$, qui engage par le fait même la responsabilité du locuteur. D'une certaine façon, affirmer que p c'est rendre public (ou du moins accessible à un tiers) le fait qu'on juge que $\mathrm{p}$.

Il est bien possible que pour juger (imaginer) que $p$, il faille "utiliser » un système symbolique quelconque, c'est-àdire évoquer une représentation mentale du fait que $p$, mais peut-on dire que le fait d'évoquer, selon tel ou tel mode, une représentation de $\mathrm{p}$ constitue un jugement que $\mathrm{p}$, au sens où l'énonciation d'une expression signifiant que p peut constituer, dans certaines conditions, une assertion que $p$ ? Alors que c'est en partie par convention qu'une énonciation a la force d'une assertion, ce n'est vraisemblablement pas par convention que l'évocation d'un état de fait (i.e. une "énonciation privée ") constitue un jugement.

Il y a néanmoins une connivence évidente entre les actes mentaux et les actes illocutoires, au point qu'on pourrait peutêtre traiter certains d'entre eux comme des variantes complémentaires de mêmes " praxèmes » et que l'explication des seconds semble devoir faire appel aux premiers. Ainsi le fait que des locuteurs-auditeurs d'un certain type aient la capacité structurelle d'effectuer certains types d'actes mentaux détermine sans doute en partie (le reste étant fonction des croyances communes aux membres de la communauté considérée) l'ensemble des actes illocutoires possibles dans toute population constituée de locu- 
teurs-auditeurs de ce type. Certains voudraient peut-être renverser la direction de l'explication et réduire les actes mentaux aux illocutions, mais un tel projet me semble voué à l'échec, dans la mesure où il devrait nier d'entrée de jeu que les animaux puissent accomplir des actes mentaux.

\subsubsection{Comprébension et utilisation}

En résumé, un système de règles d'utilisation pour un système linguistique (et une population donnée) doit déterminer, pour chaquë expression, quelle est la valeur spécifique de son énonciation dans telles ou telles circonstances. Il s'agit en d'autres termes d'expliquer comment chaque énonciation d'une expression linguistique (du système donné) est comprise en fonction du contexte d'énonciation. Les règles d'utilisation d'un système linguistique prendront par conséquent la forme de règles de compréhension des énoncés plutôt que de règles de production.

La raison en est que la connaissance de la valeur de son énoncé, et donc l'intention qu'elle soit reconnue par un destinataire éventuel, n'est qu'un des facteurs susceptibles d'intervenir dans l'explication du fait que cet énoncé ait été produit par un locuteur donné. L'énonciation d'une expression, eut aussi s'expliquer par la recherche d'autres effets que la reconnaissance de sa valeur, y compris celle d'effets dont la réalisation ne dépend pas de la reconnaissance de sa valeur. Cependant il ne suffit pas que le locuteur croie que l'énonciation d'une certaine expression dans une situation donnée produira tous les effets recherchés pour qu'il énonce cette expression dans l'intention de produire ces effets ; car il peut croire aussi que l'énonciation d'une autre expression pourrait produire les mêmes effets. Comme il n'est pas vraisemblable que l'énonciation de toute expression doive s'expliquer par la recherche d'effets dont le locuteur croit qu'aucune autre expression n'est en mesure de les produire, il y a probablement d'autres facteurs qui contribuent à expliquer l'énonciation (-token) d'une expression particulière. On peut songer soit à des critères supplémentaires que le locuteur ferait (ou pourrait faire) intervenir dans un processus de choix rationnel (simplicité, économie, etc . . .), soit à des causes qui échapperaient à la sphère du choix rationnel (habitudes, pulsions ou désirs inconscients, réflexes conditionnés, etc ...). Mais ces 
facteurs sont susceptibles d'intervenir dans l'explication de toutes les actions ou tous les types de comportement ; ils ne concernent pas spécifiquement l'utilisation des systèmes linguistiques et ne déterminent l'énonciation des expressions linguistiques que dans la mesure où elle n'est pas purement intentionnelle.

Autrement dit, dans la mesure où la production d'un énoncé n'est pas déterminée par la connaissance des règles de compréhension, elle ne concerne pas la pragmatique ; et dans la mesure où elle concerne le pragmatique, elle présuppose la connaissance des règles de compréhension. Il faut prendre garde cependant de confondre la compréhension d'un énoncé (i.e. l'identification de sa valeur) avec la compréhension des raisons de l'énonciation.

Chaque système pragmatique comprendra donc la spécification d'un ensemble de contextes d'énonciation possibles, d'un ensemble de valeurs d'énonciation possibles et d'un système de règles permettant d'associer une valeur à l'énonciation de chaque expression relativement à un contexte quelconque. Il reviendrait à la pragmatique générale pure d'expliquer les notions d'énoncé, de contexte, de valeur d'énonciation (ou illocution) et de règles de compréhension.

\subsubsection{Utilisateurs et niveaux de représentation}

La notion la plus générale que nous ayons d'un utilisateur possible pour un système linguistique est sans doute celle d'un dispositif capable de manipuler des données symboliques selon certaines règles, et la notion la plus générale que nous ayons d'un tel mécanisme est celle d'une machine de Turing. Aussi général que soit ce concept, il impose néanmoins certaines restrictions sur l'ensemble des systèmes linguistiques et des systèmes de règles d'utilisation susceptibles d'entrer dans la composition d'un système pragmatique possible. Il ne semble pas possible de maintenir que, de même que tout système syntaxique est interprétable, tout système linguistique doit pouvoir être utilisé par un certain type de dispositifs ou d'organismes sans faire violence aux intuitions les plus élémentaires concernant les conditions nécessaires de l'utilisation d'un système linguistique. L'une de ces conditions est précisément que tout utilisateur d'un système linguistique dispose d'une représentation d'une gram- 
maire et d'un ensemble de règles d'ütilisation de ce système linguistique, de façon à pouvoir identifier effectivement la valeur de chaque énonciation et le cas échéant reconnaître qu'une énonciation n'a pas de valeur déterminée). Quiconque épouserait le point de vue qu'à tout système linguistique correspond un type d'utilisateurs possibles se placerait dans la position d'avoir à expliquer, par exemple, quel genre de dispositif pourrait utiliser un langage contenant des phrases de longueur infinie. Il se peut que la tâche ne soit pas impossible, elle est en tout cas sans grand'intérêt. Mais quelles que soient, en définitive, les caractéristiques structurelles essentielles des locuteurs-auditeurs possibles, il semble indispensable de leur prêter aussi certaines propriétés «dispositionnelles», en particulier la rationalité ; et ceci vaut autant pour la pragmatique descriptive que pour la pragmatique pure.

De même que les règles grammaticales d'un système linguistique représentent la fonction qui énumère l'ensemble des expressions (sémantiquement) interprétées de ce système linguistique, on peut considérer que les règles d'utilisation d'un système pragmatique représente la fonction qui en énumère l'ensemble des énoncés (pragmatiquement) interprétés. Ainsi, de même qu'un système linguistique doit être identifié à l'ensemble de ses expressions interprétées plutôt qu'à ses règles grammaticales de même un système pragmatique sera identifié à l'ensemble de ses énoncés interprétés plutôt qu'aux règles de compréhension par lesquelles on peut le définir.

En d'autres termes, bien qu'un système linguistique ou pragmatique ne puisse être appréhendé qu'au moyen d'un certain ensemble (fini) de règles, cela ne signifie pas que le fait qu'un système linguistique ou pragmatique soit caractérisé par tel ou tel ensemble de règles doive être interprété comme une hypothèse sur la structure interne des utilisateurs. Une telle interprétation est cependant toujours possible, tant que le système linguistique ou pragmatique donné est récursivement énumérable.

Cela n'est qu'une façon de souligner que la même fonction caractéristique peut être représentée par plus d'un algorithme et qu'un système linguistique ou pragmatique ne doit pas être 
confondu avec une de ses représentations. On pourrait ajouter que chaque algorithme (ou système de règles) peut lui-même être codé de plus d'une façon dans un organisme ou une machine quelconque, et distinguer ainsi trois niveaux de modélisation des sujets linguistiques, ordonnés sur une échelle allant du général au particulier.

Au premier niveau, l'utilisateur est considéré comme un système défini uniquement comme une relation (en extension) entre des conditions d'entrée et des conditions de sortie. Au second niveau, il s'agit de définir les procédures par lesquelles l'utilisateur calcule effectivement la relation (ou plus exactement la fonction), définie au premier niveau (il faut donc que celleci satisfasse les contraintes imposées par la structure fonctionnelle interne de l'utilisateur). Au troisième niveau, on cherchera à déterminer comment les procédures identifiées au deuxième niveau sont physiquement réalisées ou codées dans l'utilisateur. Celui-ci est donc considéré au premier niveau comme une boîte noire, au deuxième niveau comme un automate abstrait et au troisième niveau comme un objet matériel. Il est clair que tout modèle du troisième type détermine un modèle du second type et un seul, qui détermine à son tour un et un seul modèle du premier type.

On peut donc s'attendre à ce que le choix d'un certain type d'utilisateurs ayant telle ou telle structure interne impose des contraintes sur l'ensemble des systèmes linguistiques ou pragmatiques dont il peut avoir la compétence ou, en d'autres termes, à ce que la structure interne d'un utilisateur contribue à expliquer certaines propriétés du système linguistique ou pragmatique dont il a la compétence. C'est précisément, me semble$\mathrm{t}$-il, la stratégie de la linguistique générative, qui identifie le second (et ultimement le troisième) niveau à celui de l'adéquation descriptive, par opposition à l'adéquation observationnelle (qui correspondrait au premier niveau). Dans cette perspective, une règle grammaticale est évaluée non seulement en raison des distributions linguistiques dont elle permet de rendre compte, mais aussi en fonction de sa « réalité psychologique ». L'adéquation explicative n'est atteinte que lorsqu'un modèle du deuxième type a pu ître " déduit " d'une description plus générale de la structure interne de ce type d'utilisateur (c'est ce que 
Chomsky appelle la grammaire universelle, qui permettrait de définir l'ensemble des grammaires possibles pour un certain type d'utilisateurs). Sans nier la légitimité ou l'intérêt du programme de Chomsky, il est incontestable (mais cela n'est pas apparu clairement à tous les linguistes) que la linguistique générative peut être considérée comme une branche de la psychologie expérimentale.

Il est douteux par contre, que le choix d'un système linguistique ou pragmatique impose des contraintes spécifiques sur la façon dont il doit être représenté dans un utilisateur que ce soit au niveau psychologique ou au niveau physique. Il est parfaitement concevable que différents utilisateurs du même système linguistique en aient des représentations différentes et même qu'ils ne puissent pas, en principe, en avoir la même représentation, sans que la possibilité de communiquer entre eux en soit affectée. Ne dirait-on pas, dans ce cas, que tous ces utilisateurs ónt la même compétence linguistique ou pragmatique ? Dire qu'un dispositif quelconque a la compétence d'un système linguistique ou pragmatique, c'est donc simplement dire qu'il a la capacité d'en reconnaître les éléments ou qu'il en connaît la fonction caractéristique, et non pas qu'il possède une représentation particulière de cette fonction caractéristique. Il ne faut pas suivre Chomsky lorsqu'il appelle les modèles du deuxième type des modèles de la compétence ; ce serait plutôt à la théorie de la performance qu'il appartiendrait d'étudier la façon dont les systèmes linguistiques ou pragmatiques sont représentés dans leurs utilisateurs.

Il n'est donc pas nécessaire, du point de vue de la sémiotique, de caractériser la structure interne spécifique des utilisateurs. Il suffit de savoir que tout utilisateur possible d'un système linguistique doit avoir une structure qui lui permette d'avoir une représentation de ce système et de ses règles d'utilisation. Chaque système pragmatique possible constitue ainsi un modèle de la compétence communicative d'une certainè classe d'utilisateurs ; mais la donnée d'un système pragmatique n'implique nullement que la structure interne des utilisateurs soit parfaitement déterminée (ni d'ailleurs l'organisation sociale de la communauté des utilisateurs). 


\subsection{Pragmatique descriptive}

Comme dans le cas de la syntaxe et de la sémantique, la tâche de la pragmatique générale descriptive serait d'identifier le plus petit ensemble de systèmes pragmatiques à contenir tous les systèmes pragmatiques empiriquement réalisés ; ou en d'autres termes, d'étudier les propriétés générales communes à tous les systèmes pragmatiques réalisés, en interprétant les résultats de la pragmatique pure comme des hypothèses empiriques.

S'il est possible, au niveau de la sémiotique pure, de faire de la syntaxe sans présupposer la sémantique, ou de la sémantique sans présupposer la pragmatique, il en va tout différemment au niveau de la sémiotique descriptive. Tout système syntaxique ne peut en effet être empiriquement manifesté que comme une composante d'un système linguistique, et tout système linguistique ne peut être réalisé que comme une composante d'un système pragmatique. En d'autres termes, l'ensemble des systèmes linguistiques réalisés, dont la grammaire descriptive doit donner une approximation, sera contenu dans celui des systèmes linguistiques utilisés et a fortiori dans celui des systèmes linguistiques utilisables. C'est dire que la seule constitution d'une pragmatique générale pure devrait permettre de réduire l'ensemble des hypothèses recevables au niveau de la grammaire descriptive.

Cela signifie aussi, et surtout, qu'il n'est pas possible, du point de vue descriptif, de séparer la pragmatique de la syntaxe et de la sémantique ; c'est-à-dire de choisir, par exemple, un certain système linguistique réalisé pour tenter ensuite d'en identifier les règles d'utilisation, car aucun système linguistique réalisé ne peut être identifié indépendamment de ses règless d'utilisation. Une hypothèse grammaticale est une hypothèse empirique incomplète tant qu'elle n'est pas assortie d'un système d'hypothèses pragmatiques. L'objectif de la sémiotique descriptive devrait donc être, d'entrée de jeu, de reconstruire des systèmes pragmatiques globaux. Cela ne concerne toutefois que la pratique de la sémiotique, et il serait présomptueux d'exclure a priori que la syntaxe soit autonome par rapport à la sémantique et à la pragmatique, et la sémantique par rapport à la pragmatique, si on doit entendre par là que les règles syntaxiques 
ne feraient pas référence aux règles sémantiques ou pragmatiques et que les règles sémantiques ne feraient pas référence aux règles pragmatiques.

C'est du moins la stratégie qui est ici recommandée ; elle se fonde sur l'idée qu'un système linguistique impose certaines contraintes sur l'ensemble des systèmes pragmatiques dont il peut faire partie, de même que, réciproquement, la façon dont un système linguistique est utilisé implique que ce système ait certaines caractéristiques. Il s'agit essentiellement de concevoir la grammaire d'une langue de telle façon qu'elle puisse contribuer à expliquer la façon dont elle est effectivement utilisée, c'est-à-dire ni plus ni moins que comme un rouage d'un système pragmatique.

Département de philosophie

Universités de Provence et du Québec à Montréal 


\section{BIBLIOGRAPHIE}

Austin, John L. (1962) : How to Do Things with Words, Oxford, Oxford Univ. Press.

Carnap, Rudolf (1942) : Introduction to Semantics, Cambridge Mass., Harvard Univ. Press.

Chomsky, Noam (1965) : Aspects of the Theory of Syntax, Cambridge Mass., M.I.T. Press.

Morris, Charles (1938)O : Foundations of the Theory of Signs, Chicago, Univ. of Chicago Press. 